\title{
Real and Complex Rank for Real Symmetric Tensors with Low Ranks
}

\author{
Edoardo Ballico ${ }^{1}$ and Alessandra Bernardi ${ }^{2}$ \\ ${ }^{1}$ Department of Mathematics, University of Trento, Povo, 38123 Trento, Italy \\ ${ }^{2}$ Department of Mathematics “Giuseppe Peano," University of Turin, 10123 Turin, Italy
}

Correspondence should be addressed to Alessandra Bernardi; alessandra.bernardi@unito.it

Received 20 November 2012; Revised 5 February 2013; Accepted 5 February 2013

Academic Editor: Ricardo L. Soto

Copyright (C) 2013 E. Ballico and A. Bernardi. This is an open access article distributed under the Creative Commons Attribution License, which permits unrestricted use, distribution, and reproduction in any medium, provided the original work is properly cited.

\begin{abstract}
We study the case of a real homogeneous polynomial $P$ whose minimal real and complex decompositions in terms of powers of linear forms are different. We prove that if the sum of the complex and the real ranks of $P$ is at most $3 \operatorname{deg}(P)-1$, then the difference of the two decompositions is completely determined either on a line or on a conic or two disjoint lines.
\end{abstract}

\section{Introduction}

The problem of decomposing a tensor into a minimal sum of rank-1 terms is raising interest and attention from many applied areas as signal processing for telecommunications [1], independent component analysis [2], complexity of matrix multiplication [3], complexity problem of $P$ versus NP [4], quantum physics [5, 6], and phylogenetics [7]. The particular instance in which the tensor is symmetric and hence representable by a homogeneous polynomial is one of the most studied and developed ones (cf. [8] and references therein). In this last case, we say that the rank of a homogeneous polynomial $P$ of degree $d$ is the minimum integer $r$ needed to write it as a linear combination of pure powers of linear forms $L_{1}, \ldots, L_{r}$ :

$$
P=c_{1} L_{1}^{d}+\cdots+c_{d} L_{r}^{d},
$$

with $c_{i} \neq 0$. Most of the papers concerning the abstract theory of the symmetric tensor rank require the base field to be algebraically closed. In this case, we may take $c_{i}=1$ for all $i$ without loss of generality. However, for the applications, it is very important to consider the case of real polynomials and look at their real decomposition. Namely, one can study separately the case in which the linear forms appearing in (1) are complex or real. In the real case we may take $c_{i}=1$ for all $i$ if $d$ is odd, while we take $c_{i} \in\{-1,1\}$ if $d$ is even. When we look for a minimal complex (resp., real) decomposition as in
(1), we say that we are computing the complex symmetric rank (resp., real symmetric rank) of $P$ and we will indicate it $r_{\mathbb{C}}(P)$ (resp., $r_{\mathbb{R}}(P)$ ). Obviously

$$
r_{\mathbb{C}}(P) \leq r_{\mathbb{R}}(P),
$$

and in many cases such an equality is strict.

In [9] Comon and Ottaviani studied the real case for bivariate symmetric tensors. Even in this case, there are many open conjectures, and, up to now, few cases are completely settled [9-12].

In this paper, we want to study the relation between $r_{\mathbb{C}}(P)$ and $r_{\mathbb{R}}(P)$ in the special circumstance in which $r_{\mathbb{C}}(P)<$ $r_{\mathbb{R}}(P)$. In particular, we will show that in a certain range (say, $\left.r_{\mathbb{C}}(P)+r_{\mathbb{R}}(P) \leq 3 \operatorname{deg}(P)-1\right)$, all homogeneous polynomials $P$ of that degree with $r_{\mathbb{R}}(P) \neq r_{\mathbb{C}}(P)$ are characterized by the existence of a curve with the property that the sets evincing the real and the complex ranks coincide out of it (see Theorem 1 for the precise statement). More precisely, let $P \in S^{d} \mathbb{R}^{m+1}$ be a real homogeneous polynomial of degree $d$ in $m+1$ variables such that $r_{\mathbb{C}}(P)<r_{\mathbb{R}}(P)$ and $r_{\mathbb{C}}(P)+r_{\mathbb{R}}(P) \leq$ $3 \operatorname{deg}(P)-1$; therefore, its real and complex decomposition are

$$
\begin{gathered}
P=a_{1} L_{1}^{d}+\cdots+a_{k} L_{k}^{d}+c_{1} M_{1}^{d}+\cdots+c_{s} M_{s}^{d}, \\
P=N_{1}^{d}+\cdots+N_{h}^{d}+c_{1} M_{1}^{d}+\cdots+c_{s} M_{s}^{d},
\end{gathered}
$$


respectively, with $k>h, a_{i} \in\{-1,1\}, c_{j} \in\{-1,1\}$, $M_{1}, \ldots, M_{s} \in S^{1} \mathbb{R}^{m+1}, h+k>d+2$. Moreover, there exists a curve $C^{l} \subset \mathbb{P}^{m}$ such that $\left[L_{1}\right], \ldots,\left[L_{k}\right],\left[N_{1}\right], \ldots,\left[N_{h}\right]$ "depends only from the variables of $C^{\prime \prime}$ and $C^{\prime}$ is either a line or a reduced conic or a disjoint union of two lines. If $C^{\prime}$ is a line (item (a) in Theorem 1 ) then both the $L_{i}$ 's and the $N_{i}$ 's are linear forms in the same two "variables." If $C^{\prime}$ is a conic, then $L_{i}$ 's and $N_{i}$ 's depend on 3 "variables" and their projectivizations lie on $C^{\prime}$. See item (c) of Theorem 1 for the geometric interpretation of the reduction of $L_{1}, \ldots, L_{k}$ and $N_{1}, \ldots, N_{h}$ to bivariate forms involved with $C^{\prime}$ when $C^{\prime}=$ $l \sqcup r$ is a disjoint union of two lines $l$ and $r$ (we have two sets of bivariate forms, one for the variables of $l$ and one for the variables of $r$ ).

\section{Notation and Statements}

Before giving the precise statement of Theorem 1 we need to introduce the main algebraic geometric tools that we will use all along the paper.

Let $v_{d}: \mathbb{P}^{m} \rightarrow \mathbb{P}^{N}, N:=\left(\begin{array}{c}m+d \\ d\end{array}\right)-1$, denote the degree $d$ Veronese embedding of $\mathbb{P}^{m}$ (say, defined over $\mathbb{C}$ ). Set $X_{m, d}:=v_{d}\left(\mathbb{P}^{m}\right)$. For any $P \in \mathbb{P}^{N}$, the symmetric rank or symmetric tensor rank or, just, the rank $r_{\mathbb{C}}(P)$ of $P$ is the minimal cardinality of a finite set $S \subset \mathbb{P}^{m}(\mathbb{C})$ such that $P \in\left\langle v_{d}(S)\right\rangle$, where \langle\rangle denote the linear span (here the linear span is with respect to complex coefficients), and we will say that $S$ evinces $r_{\mathbb{C}}(P)$. Notice that the Veronese embedding $v_{d}$ is defined over $\mathbb{R}$, that is, $v_{d}\left(\mathbb{P}^{m}(\mathbb{R})\right) \subset \mathbb{P}^{N}(\mathbb{R})$. For each $P \in \mathbb{P}^{N}(\mathbb{R})$ the real symmetric rank $r_{\mathbb{R}}(P)$ of $P$ is the minimal cardinality of a finite set $S \subset \mathbb{P}^{m}(\mathbb{R})$ such that $P \in\left\langle v_{d}(S)\right\rangle_{\mathbb{R}}$, where \langle\rangle$_{\mathbb{R}}$ means the linear span with real coefficients, and we will say that $S$ evinces $r_{\mathbb{R}}(P)$. The integer $r_{\mathbb{R}}(P)$ is well defined because $v_{d}\left(\mathbb{P}^{m}(\mathbb{R})\right)$ spans $\mathbb{P}^{N}(\mathbb{R})$.

Let us fix some notation: if $C \subset \mathbb{P}^{m}$ is either a curve or a subspace and $S \subset \mathbb{P}^{m}$ is a finite set, we will use the following abbreviations:

$$
\begin{gathered}
S_{C}:=S \cap C, \\
S_{\widehat{C}}:=S \backslash(S \cap C) .
\end{gathered}
$$

Theorem 1. Let $P \in \mathbb{P}^{N}(\mathbb{R})$ be such that $r_{\mathbb{C}}(P)+r_{\mathbb{R}}(P) \leq$ $3 d-1$ and $r_{\mathbb{C}}(P) \neq r_{\mathbb{R}}(P)$. Fix any set $S_{\mathbb{C}} \subset \mathbb{P}^{m}(\mathbb{C})$ and $S_{\mathbb{R}} \subset$ $\mathbb{P}^{m}(\mathbb{R})$ evincing $r_{\mathbb{C}}(P)$ and $r_{\mathbb{R}}(P)$, respectively. Then one of the following cases (a), (b), and (c) occurs.

(a) There is a line $l \subset \mathbb{P}^{m}$ defined over $\mathbb{R}$ and with the following properties:

(i) $S_{\mathbb{C}}$ and $S_{\mathbb{R}}$ coincide out of the line l in a set $S_{l}$ :

$$
S_{\mathbb{C}} \backslash S_{\mathbb{C}} \cap l=S_{\mathbb{R}} \backslash S_{\mathbb{R}} \cap l=: S_{\bar{l}} ;
$$

(ii) there is a point $P_{l} \in\left\langle v_{d}\left(S_{\mathbb{C}, l}\right)\right\rangle \cap\left\langle v_{d}\left(S_{\mathbb{R}, l}\right)\right\rangle$ such that $S_{\mathbb{C}, l}$ evinces $r_{\mathbb{C}}\left(P_{l}\right)$ and $S_{\mathbb{R}, l}$ evinces $r_{\mathbb{R}}\left(P_{l}\right)$;

(iii) $\sharp\left(S_{\mathbb{C}, l} \cup S_{\mathbb{R}, l}\right) \geq d+2$ and $\sharp\left(S_{\mathbb{C}, l}\right)<\sharp\left(S_{\mathbb{R}, l}\right)$.

(b) There is a conic $C \subset \mathbb{P}^{m}$ defined over $\mathbb{R}$ and with the following properties: (i) $S_{\mathbb{C}}$ and $S_{\mathbb{R}}$ coincide out of the conic $C$ in a set $S_{\widehat{C}}$ :

$$
S_{\mathbb{C}} \backslash S_{\mathbb{C}, C}=S_{\mathbb{R}} \backslash S_{\mathbb{R}} \cap C=: S_{\widehat{C}} ;
$$

(ii) there is a point $P_{C} \in\left\langle v_{d}\left(S_{\mathbb{C}, C}\right)\right\rangle \cap\left\langle v_{d}\left(S_{\mathbb{R}, C}\right)\right\rangle$ such that $S_{\mathbb{C}, C}$ evinces $r_{\mathbb{C}}\left(P_{C}\right)$ and $S_{\mathbb{R}, C}$ evinces $r_{\mathbb{R}}\left(P_{C}\right)$;

(iii) $\sharp\left(S_{\mathbb{C}, C} \cup S_{\mathbb{R}, C}\right) \geq 2 d+2$ and $\sharp\left(S_{\mathbb{C}, C}\right)<\sharp\left(S_{\mathbb{R}, C}\right)$;

(iv) if $C$ is reducible, say $C=l_{1} \cup l_{2}$ with $Q=l_{1} \cap l_{2}$, then $\sharp\left(\left(S_{\mathbb{C}} \cup S_{\mathbb{R}}\right) \cap\left(l_{i} \backslash Q\right)\right) \geq d+1$ for $i \in\{1,2\}$.

(c) $m \geq 3$ and there are 2 disjoint lines $l, r \subset \mathbb{P}^{m}$ defined over $\mathbb{R}$ with the following properties:

(i) $S_{\mathbb{C}}$ and $S_{\mathbb{R}}$ coincide out of the union $\Gamma:=l \cup r$ in a set $S_{\widehat{\Gamma}}$ :

$S_{\mathbb{C}} \backslash S_{\mathbb{C}} \cap(l \cup r)=S_{\mathbb{R}} \backslash S_{\mathbb{R}} \cap(l \cup r):=S_{\widehat{\Gamma}} ;$

(ii) $\sharp\left(S_{\mathbb{C}, l} \cup S_{\mathbb{R}, l}\right) \geq d+2$ and $\sharp\left(S_{\mathbb{C}, r} \cup S_{\mathbb{R}, r}\right) \geq d+2$;

(iii) the set $\left\langle v_{d}\left(S_{\widehat{\Gamma}}\right)\right\rangle \cap\left\langle v_{d}(\Gamma)\right\rangle$ is a single point, $O_{\Gamma} \in$ $\mathbb{P}^{N}(\mathbb{R})$ :

$S_{\mathbb{C}, \Gamma}$ evinces $r_{\mathbb{C}}\left(O_{\Gamma}\right)$ and $S_{\mathbb{R}, \Gamma}$ evinces $O_{\Gamma}$;

(iv) the set $\left\langle\left\{O_{\Gamma}\right\} \cup v_{d}(l)\right\rangle \cap\left\langle v_{d}(l)\right\rangle$ (resp., $\left\langle\left\{O_{\Gamma}\right\} \cup\right.$ $\left.\left.v_{d}(r)\right\rangle \cap\left\langle v_{d}(r)\right\rangle\right)$ is formed by a unique point $O_{l} \in \mathbb{P}^{N}(\mathbb{R})\left(\right.$ resp. $\left.O_{r} \in \mathbb{P}^{N}(\mathbb{R})\right)$ :

$S_{\mathbb{C}, l}\left(\right.$ resp., $\left.S_{\mathbb{C}, r}\right)$ evinces $r_{\mathbb{C}}\left(O_{l}\right)$ (resp., $r_{\mathbb{C}}\left(O_{r}\right)$ );

$S_{\mathbb{R}, l}\left(\right.$ resp., $\left.S_{\mathbb{R}, r}\right)$ evinces $r_{\mathbb{R}}\left(O_{l}\right)\left(\right.$ resp., $\left.r_{\mathbb{R}}\left(O_{r}\right)\right)$.

\section{The Proof}

Remark 2. Let $S \subset \mathbb{P}^{N}(\mathbb{R})$. It will be noteworthy in the sequel that $S$ can be used to span both a real space $\langle S\rangle_{\mathbb{R}} \subset \mathbb{P}^{N}(\mathbb{R})$ and a complex space $\langle S\rangle_{\mathbb{C}} \subset \mathbb{P}^{N}(\mathbb{C})$ of the same dimension and $\langle S\rangle_{\mathbb{C}} \cap \mathbb{P}^{N}(\mathbb{R})=\langle S\rangle_{\mathbb{R}}$. In the following, we will always use \langle\rangle to denote \langle\rangle$_{\mathbb{C}}$.

Remark 3. Fix $P \in \mathbb{P}^{N}$ and a finite set $S \subset \mathbb{P}^{N}$ such that $S$ evinces $r_{\mathbb{C}}(P)$. Fix any $E \varsubsetneqq S$. Then the set $\langle\{P\} \cup E\rangle \cap\langle S \backslash E\rangle$ is a single point (call it $P_{1}$ ) and $S \backslash E$ evinces $r_{\mathbb{C}}\left(P_{1}\right)$. Now assume $P \in \mathbb{P}^{N}(\mathbb{R})$ and $S \subset \mathbb{P}^{N}(\mathbb{R})$. Then $P_{1} \in \mathbb{P}^{N}(\mathbb{R})$. If $S$ evinces $r_{\mathbb{R}}(P)$, then $S \backslash E$ evinces $r_{\mathbb{R}}\left(P_{1}\right)$.

Lemma 4. Let $C \subset \mathbb{P}^{m}$ be a reduced curve of degree $t$ with $t=1,2$. Fix finite sets $A, B \subset \mathbb{P}^{m}$. Fix an integer $d>t$ such that

$$
h^{1}\left(\mathscr{I}_{(A \cup B)_{\hat{C}}}(d-t)\right)=0 .
$$

Assume the existence of $P \in\left\langle v_{d}(A)\right\rangle \cap\left\langle v_{d}(B)\right\rangle$ and $P \notin$ $\left\langle v_{d}\left(S^{\prime}\right)\right\rangle$ for any $S^{\prime} \varsubsetneqq A$ and any $S^{\prime} \varsubsetneqq B$. Then

$$
A_{\widehat{\mathrm{C}}}=B_{\widehat{\mathrm{C}}} .
$$

Proof. The case $t=1$ is [13, Lemma 8]. If $t=2$, then either $C$ is a conic or $m \geq 3$ and $C$ is a disjoint union of 2 lines. In both cases, we have $h^{0}\left(\mathscr{I}_{C}(t)\right)>0$, and the linear system $\left|\mathscr{I}_{C}(t)\right|$ has no base points outside $C$. Since $A \cup B$ is a finite set, there is $M \in\left|\mathscr{I}_{C}(t)\right|$ such that $M \cap(A \cup B)=C \cap(A \cup B)$. 
Look at the following residual exact sequence (also called the Castelnuovos exact sequence):

$$
\begin{aligned}
0 \longrightarrow \mathscr{I}_{(A \cup B)_{\tilde{C}}}(d-t) \longrightarrow \mathscr{I}_{A \cup B}(d) \\
\longrightarrow \mathscr{I}_{(A \cup B) \cap M, M}(d) \longrightarrow 0
\end{aligned}
$$

We can now repeat the same proof of [13, Lemma 8] but starting with (10) instead of the exact sequence used there (cf. first displayed formula in the proof of [13, Lemma 8]).

We will therefore get $A_{\widehat{M}}=B_{\widehat{M}}$. Now, since $M \cap(A \cup B)=$ $C \cap(A \cup B)$, we are done.

We are now going to prove Theorem 1.

Proof of Theorem 1. Fix $P \in \mathbb{P}^{N}(\mathbb{R})$ such that $r_{\mathbb{C}}(P)+r_{\mathbb{R}}(P) \leq$ $3 d-1$ and $r_{\mathbb{C}}(P) \neq r_{\mathbb{R}}(P)$.

Fix any set $S_{\mathbb{C}} \subset \mathbb{P}^{m}(\mathbb{C})$ evincing $r_{\mathbb{C}}(P)$ and any $S_{\mathbb{R}} \subset$ $\mathbb{P}^{m}(\mathbb{R})$ evincing $r_{\mathbb{R}}(P)$.

By applying [14], Lemma 4, we immediately get that

$$
h^{1}\left(\mathscr{I}_{S_{\mathbb{C}} \cup S_{\mathbb{R}}}(d)\right)>0 .
$$

Since $\sharp\left(S_{\mathbb{C}}\right)+\sharp\left(S_{\mathbb{R}}\right) \leq 3 d-1$, either there is a line $l \subset \mathbb{P}^{m}$ such that $\sharp\left(S_{\mathbb{C}, l} \cup S_{\mathbb{R}, l}\right) \geq d+2$ or there is a conic $C$ such that $\sharp\left(S_{\mathbb{C}, C} \cup S_{\mathbb{R}, C}\right) \geq 2 d+2$ (Theorem 3.8, [15]). We are going to study separately these two cases in items (1) and (10) below.

(1) In this step, we assume the existence of a line $l \subset \mathbb{P}^{m}$ such that

$$
\sharp\left(S_{\mathbb{C}, l} \cup S_{\mathbb{R}, l}\right) \geq d+2 .
$$

This hypothesis, together with $r_{\mathbb{R}}(P) \neq r_{\mathbb{C}}(P)$, immediately implies property (a)-(iii) of the statement of the theorem.

We are now going to distinguish the case $h^{1}\left(\mathscr{I}_{S_{\mathbb{C}} \cup S_{\mathbb{R}}}(d-\right.$ $1))=0($ item $(1.1)$ below $)$ from the case $h^{1}\left(\mathscr{I}_{S_{\mathcal{C}} \cup S_{\mathbb{R}}}(d-1)\right)>0$ (item (1.2) below).

(1.1) Assume $h^{1}\left(\mathscr{I}_{S_{\mathbb{C}} \cup S_{\mathbb{R}}}(d-1)\right)=0$.

First of all, observe that the line $l \subset \mathbb{P}^{m}$ is well defined over $\mathbb{R}$ since it contains at least 2 points of $S_{\mathbb{R}}$ (Remark 2 ). Then, by Lemma 4 , we have that $S_{\mathbb{C}}$ and $S_{\mathbb{R}}$ have to coincide out of the line $l$ :

$$
S_{\mathbb{C}} \backslash S_{\mathbb{C}, l}=S_{\mathbb{R}} \backslash S_{\mathbb{R}, l}:=S_{\hat{l}},
$$

and this proves (a)-(i) of the statement of the theorem in this case (1.1).

The fact that $\sharp\left(S_{\mathbb{C}}\right)<\sharp\left(S_{\mathbb{R}}\right)$ implies that $\sharp\left(S_{\mathbb{R}, l}\right)>(d+2) / 2$ and $\sharp\left(S_{\tilde{l}}\right) \leq d$; hence, $h^{1}\left(\mathscr{I}_{S_{\bar{l}} \cup l}(d)\right)=0$.

Therefore, we have that $\operatorname{dim}\left(\left\langle v_{d}\left(S_{\hat{l}} \cup l\right)\right\rangle\right)=\sharp\left(S_{\hat{l}}\right)+d+$ $1, \operatorname{dim}\left(\left\langle\nu_{d}\left(S_{\tilde{l}}\right)\right\rangle\right)=\sharp\left(S_{\bar{l}}\right)-1$ and $\operatorname{dim}\left(\left\langle\nu_{d}(l)\right\rangle\right)=d+1$, and Grassmann's formula gives $\left\langle\nu_{d}\left(S_{\uparrow}\right)\right\rangle \cap\left\langle\nu_{d}(l)\right\rangle=\emptyset$.

Since $P \in\left\langle\nu_{d}\left(S_{\bar{l}} \cup S_{\mathbb{C}}\right)\right\rangle$ and $S_{\mathbb{C}, l} \subset l$, the set $\left\langle\nu_{d}\left(S_{\bar{l}}\right) \cup\{P\}\right\rangle \cap$ $\left\langle\nu_{d}(l)\right\rangle$ is a single point, $P_{l} \in \mathbb{P}^{N}(\mathbb{R})$.

Since $P \in\left\langle\nu_{d}\left(S_{\mathbb{C}}\right)\right\rangle$ and $P \notin\left\langle\nu_{d}\left(S_{\mathbb{C}}^{\prime}\right)\right\rangle$ for any $S^{\prime} \varsubsetneqq S_{\mathbb{C}}$, the set $\left\langle v_{d}\left(S_{\tilde{l}}\right) \cup\{P\}\right\rangle \cap\left\langle v_{d}\left(S_{\mathbb{C}, l}\right)\right\rangle$ is a single point, $P_{C}$ (Remark 3 ).

Then obviously

$$
P_{C}=P_{l} \in \mathbb{P}^{N}(\mathbb{R}) .
$$

Since $S_{\mathbb{C}}$ evinces $r_{\mathbb{C}}(P)$, then $S_{\mathbb{C}, l}$ evinces $P_{l}$ (Remark 3 ). In the same way, we see that $\left\langle v_{d}\left(S_{\tilde{l}}\right) \cup\{P\}\right\rangle \cap\left\langle v_{d}\left(S_{\mathbb{R}, l}\right)\right\rangle=\left\{P_{l}\right\}$ and that $S_{\mathbb{R}, l}$ evinces $r_{\mathbb{R}}\left(P_{l}\right)$. This proves (a)-(ii) of Theorem 1 in this case (1.1).

(1.2) Assume $h^{1}\left(\mathscr{I}_{S_{\mathbb{C}} \cup S_{\mathbb{R}}}(d-1)\right)>0$.

First of all, observe that there exists a line $r \subset \mathbb{P}^{m}$ such that $\sharp\left(r \cap\left(S_{\mathbb{C}} \cup S_{\mathbb{R}}\right)_{\hat{l}}\right) \geq d+1$, because $\sharp\left(S_{\mathbb{C}} \cup S_{\mathbb{R}}\right)_{\hat{l}} \leq 3 d-1-d-2 \leq$ $2(d-1)+1$.

By the same reason, if we write $C:=l \cup r$, we get that $\sharp\left(S_{\mathbb{C}} \cup S_{\mathbb{R}}\right)_{\widehat{C}} \leq 3 d-1-d-2-d-1 \leq d-2$ and hence $h^{1}\left(\mathscr{I}_{\left(S_{\mathbb{C}} \cup S_{\mathbb{R}}\right)_{\hat{C}}}(d-2)\right)=0$ (e.g., by [16], Lemma 34, or by [15], Theorem 3.8). Lemma 4 gives

$$
S_{\mathbb{C}, \widehat{C}}=S_{\mathbb{R}, \widehat{C}}
$$

Assume for the moment $l \cap r \neq \emptyset$. In this case, Remark 3 indicates that we can consider case (b) of the statement of the theorem. Therefore, (15) proves (b)-(i) in the case that the conic $C$ in (b) in the statement of the theorem is reduced. Moreover, condition (b)-(iv) is satisfied because $\sharp\left(r \cap\left(S_{\mathbb{C}} \cup\right.\right.$ $\left.\left.S_{\mathbb{R}}\right)_{\bar{l}}\right) \geq d+1$.

Now assume $l \cap r=\emptyset$. We will check that we are in case (a) with respect to the line $l$ if $\sharp\left(S_{\mathbb{C}, r} \cup S_{\mathbb{R}, r}\right)=d+1$, while we are in case (c) with respect to the lines $l$ and $r$ if $\sharp\left(S_{\mathbb{C}, r} \cup S_{\mathbb{R}, r}\right) \geq d+2$, and the case $\sharp\left(S_{\mathbb{C}, l} \cup S_{\mathbb{R}, l}\right)=\sharp\left(S_{\mathbb{C}, r} \cup S_{\mathbb{R}, r}\right)=d+1$ cannot occur.

Set $\Gamma:=l \cup r$. Since $r \cap l=\emptyset$, we have $\operatorname{dim}\langle\Gamma\rangle=3$ and hence $m \geq 3$.

Assume for the moment $m \geq 4$. Hence, $\sharp\left(S_{\mathbb{C}} \cup S_{\mathbb{R}}\right)_{\widehat{\langle\Gamma\rangle}} \leq d$ and $h^{1}\left(\mathscr{I}_{\left(S_{\mathbb{C}} \cup S_{\mathbb{R}}\right)_{\overline{\langle\Gamma\rangle}}}(d-1)\right)=0$. Therefore

$$
S_{\mathbb{C}, \widehat{\langle\Gamma\rangle}}=S_{\mathbb{R}, \widehat{\langle\Gamma\rangle}},
$$

and the set $\left\langle\{P\} \cup \nu_{d}\left(S_{\mathbb{C}, \widehat{\langle\Gamma\rangle}}\right)\right\rangle \cap\left\langle\nu_{d}(\langle\Gamma\rangle)\right\rangle$ is a single real point:

$$
O:=\left\langle\{P\} \cup v_{d}\left(S_{\mathbb{C}, \widehat{\langle\Gamma\rangle}}\right)\right\rangle \cap\left\langle v_{d}(\langle\Gamma\rangle)\right\rangle \in \mathbb{P}^{N}(\mathbb{R}),
$$

$S_{\mathbb{C}, \Gamma}$ evinces $r_{\mathbb{C}}(O)$ and $S_{\mathbb{R}, \Gamma}$ evinces $r_{\mathbb{R}}(O)$. Now, (16) implies that if we are either in case (a) or in case (c) of the theorem, we can simply study what happens at $S_{\mathbb{C},\langle\Gamma\rangle}$ and at $S_{\mathbb{R},\langle\Gamma\rangle}$, which means that we can reduce our study to the case $m=3$, since $\langle\Gamma\rangle=\mathbb{P}^{3}$.

Until step (2) below, we will assume $m=3$.

The linear system $\left|\mathscr{I}_{\Gamma}(2)\right|$ on $\langle\Gamma\rangle$ has no base points outside $\Gamma$ itself. Since $S_{\mathbb{C}} \cup S_{\mathbb{R}}$ is finite, there is a smooth quadric surface $W$ containing $\Gamma$ such that

$$
S_{\mathbb{C}, W} \cup S_{\mathbb{R}, W}=S_{\mathbb{C}, \Gamma} \cup S_{\mathbb{R}, \Gamma}
$$

Moreover, such a $W$ can be found among the real smooth quadrics, since $l$ and $r$ are real lines.

Since $\sharp\left(S_{\mathbb{C},\langle\Gamma\rangle} \cup S_{\mathbb{R},\langle\Gamma\rangle}\right)_{\widehat{W}} \leq d-1$, we have $h^{1}\left(\mathscr{I}_{\left(S_{\mathbb{C}} \cup S_{\mathbb{R}}\right)_{\widehat{W}}}(d-\right.$ $2))=0$. Hence, Lemma 4 applied to the point $O$ defined in (17) gives

$$
\left(S_{\mathbb{C},\langle\Gamma\rangle}\right)_{\widehat{W}}=\left(S_{\mathbb{R},\langle\Gamma\rangle}\right)_{\widehat{W}},
$$

$\left\langle\{O\} \cup \nu_{d}\left(S_{\mathbb{C},\langle\Gamma,\rangle}\right)_{\widehat{W}}\right\rangle \cap\left\langle v_{d}(W)\right\rangle$ is a single real point,

$$
O^{\prime}=\left\langle\{O\} \cup v_{d}\left(S_{\mathbb{C},\langle\Gamma,\rangle}\right)_{\widehat{W}}\right\rangle \cap\left\langle\nu_{d}(W)\right\rangle \in \mathbb{P}^{N}(\mathbb{R}),
$$


and $S_{\mathbb{C}, W}$ evinces $r_{\mathbb{C}}\left(\mathrm{O}^{\prime}\right)$. If $\left(\mathrm{O}^{\prime}, S_{\mathbb{C}, W}, S_{\mathbb{R}, W}\right)$ is either as in case (a) or in case (c) of the statement of the theorem, then $\left(O, S_{\mathbb{C},\langle\Gamma\rangle}, S_{\mathbb{R},\langle\Gamma\rangle}\right)$ is in the same case. Consider the system $|(1,0)|$ of lines on the smooth quadric surface $W$ containing $\Gamma$. We have that $h^{1}\left(W, \mathcal{O}_{W}(d-2, d)\right)=0$, and hence the restriction map $H^{0}\left(W, \mathcal{O}_{W}(d)\right) \rightarrow H^{0}\left(\Gamma, \mathcal{O}_{\Gamma}(d)\right)$ is surjective. Therefore,

$$
\begin{aligned}
h^{1}\left(W, \mathscr{I}_{W \cap\left(S_{\mathbb{C}} \cup S_{\mathbb{R}}\right)}(d)\right)= & h^{1}\left(l, \mathscr{I}_{l \cap\left(S_{\mathbb{C}} \cup S_{\mathbb{R}}\right), l}(d)\right) \\
& +h^{1}\left(r, \mathscr{I}_{r \cap\left(S_{\mathbb{C}} \cup S_{\mathbb{R}}\right), r}(d)\right) .
\end{aligned}
$$

Now, this last equality, together with the facts that $v_{d}\left(S_{\mathbb{C}, W}\right)$ and $v_{d}\left(S_{\mathbb{R}, W}\right)$ are linearly independent and $\left(S_{\mathbb{C}} \cup S_{\mathbb{R}}\right)_{W} \subset \Gamma$, gives

$$
\begin{aligned}
\operatorname{dim}( & \left.\left\langle v_{d}\left(S_{\mathbb{C}, W}\right)\right\rangle \cap\left\langle v_{d}\left(S_{\mathbb{R}, W}\right)\right\rangle\right) \\
= & \sharp\left(S_{\mathbb{C}} \cap S_{\mathbb{R}}\right)_{l}+\sharp\left(S_{\mathbb{C}} \cap S_{\mathbb{R}}\right)_{r} \\
& +h^{1}\left(l, \mathscr{I}_{\ln \left(S_{\mathbb{C}} \cup S_{\mathbb{R}}\right), l}(d)\right) \\
& +h^{1}\left(r, \mathscr{I}_{r \cap\left(S_{\mathbb{C}} \cup S_{\mathbb{R}}\right), r}(d)\right) .
\end{aligned}
$$

(1.2.1) Observe that (22) implies that the case $\sharp\left(S_{\mathbb{C}} \cup S_{\mathbb{R}}\right)_{r}=$ $\sharp\left(S_{\mathbb{C}} \cup S_{\mathbb{R}}\right)_{l}=d+1$ cannot happen because there is no contribution from $h^{1}\left(l, \mathscr{I}_{\ln \left(S_{\mathbb{C}} \cup S_{\mathbb{R}}\right), l}(d)\right)+h^{1}\left(r, \mathscr{I}_{r \cap\left(S_{\mathbb{C}} \cup S_{\mathbb{R}}\right), r}(d)\right)$ since both terms, in this case, are equal to 0 . So, we can assume that at least $\sharp\left(S_{\mathbb{C}} \cup S_{\mathbb{R}}\right)_{l}>d+1$.

(1.2.2) Assume $\sharp\left(S_{\mathbb{C}} \cup S_{\mathbb{R}}\right)_{r}=d+1$ and $\sharp\left(S_{\mathbb{C}} \cup S_{\mathbb{R}}\right)_{l}>d+1$.

To prove that we are in case (a) with respect to $l$ it is sufficient to prove $S_{\mathbb{C}, r}=S_{\mathbb{R}, r}$.

We have $\operatorname{dim}\left\langle v_{d}\left(S_{\mathbb{C}} \cup S_{\mathbb{R}}\right)\right\rangle=\sharp\left(S_{\mathbb{C}} \cup S_{\mathbb{R}}\right)-1$ - $h^{1}\left(\mathscr{I}_{S_{\mathbb{C}} \cup S_{\mathbb{R}}}(d)\right)$. Since $v_{d}\left(S_{\mathbb{C}}\right)$ and $v_{d}\left(S_{\mathbb{R}}\right)$ are linearly independent, $\sharp\left(S_{\mathbb{C}} \cup S_{\mathbb{R}}\right)=\sharp\left(S_{\mathbb{C}}\right)+\sharp\left(S_{\mathbb{R}}\right)-\sharp\left(S_{\mathbb{C}} \cap S_{\mathbb{R}}\right), \sharp\left(S_{\mathbb{C}, l} \cup\right.$ $\left.S_{\mathbb{R}, l}\right)=\sharp\left(S_{\mathbb{C}, l}\right)+\sharp\left(S_{\mathbb{R}, l}\right)-\sharp\left(S_{\mathbb{C}, l} \cap S_{\mathbb{R}, l}\right)$ and $h^{1}\left(\mathscr{I}_{S_{\mathbb{C}} \cup S_{\mathbb{R}}}(d)\right)=$ $h^{1}\left(l, \mathscr{I}_{S_{\mathbb{C},} \cup S_{\mathbb{R}, l}}\right)$, Grassmann's formula gives that $\left\langle v_{d}\left(S_{\mathbb{C}}\right)\right\rangle \cap$ $\left\langle\nu_{d}\left(S_{\mathbb{R}}\right)\right\rangle$ is generated by $\left\langle\nu_{d}\left(S_{\mathbb{C}, l}\right)\right\rangle \cap\left\langle\nu_{d}\left(S_{\mathbb{R}, l}\right)\right\rangle$. Since $P \notin$ $\left\langle\nu_{d}\left(S^{\prime}\right)\right\rangle$ for any $S^{\prime} \varsubsetneqq S_{\mathbb{C}}$, we get $S_{\mathbb{C}}=S_{\mathbb{C}, l} \cup\left(S_{\mathbb{C}} \cap S_{\mathbb{R}}\right)_{\hat{l}}$, that is, $S_{\widehat{l}}=S_{\mathbb{R}} \backslash S_{\mathbb{C}, l}$. Hence, we can consider case (a), and (15) proves property (a)-(i) also for the case (1.2) that we are treating. The point $P_{l}$ that we need to get (a)-(ii) can be identified with the point $O^{\prime}$ defined in (20) while (a)-(iii) comes from our hypotheses.

This gives all cases (a) of Theorem 1 .

(1.2.3) Assume that both $\sharp\left(S_{\mathbb{C}} \cup S_{\mathbb{R}}\right)_{r} \geq d+2$ and $\sharp\left(S_{\mathbb{C}} \cup\right.$ $\left.S_{\mathbb{R}}\right)_{l} \geq d+2$.

We need to prove that we are in case (c). Recall that $S_{\mathbb{C}, \widehat{\Gamma}}=$ $S_{\mathbb{R}, \hat{\Gamma}}$ and that $h^{1}\left(\mathscr{I}_{S_{\mathbb{C}, \hat{\Gamma}} \cup \Gamma}(d)\right)=0$. The latter equality implies, as in Remark 3, that $\left\langle\{P\} \cup \nu_{d}\left(S_{\mathbb{C}, \Gamma}\right)\right\rangle \cap\left\langle\nu_{d}(\Gamma)\right\rangle$ is a single real point $O_{1}$, that $S_{\mathbb{C}, \Gamma}$ evinces $r_{\mathbb{C}}\left(O_{1}\right)$, and that $S_{\mathbb{R}, \Gamma}$ evinces $O_{1}$. Now $O_{1}$ plays the role of $O_{\Gamma}$ of case (c)-(iii) in Theorem 1.

Since $\left\langle\nu_{d}(l)\right\rangle \cap\left\langle\nu_{d}(r)\right\rangle=\emptyset$ and $O_{1} \in\left\langle\nu_{d}(\Gamma)\right\rangle$, the sets $\left\langle\left\{O_{1}\right\} \cup v_{d}(l)\right\rangle \cap\left\langle v_{d}(l)\right\rangle$ (resp., $\left.\left\langle\left\{O_{1}\right\} \cup v_{d}(r)\right\rangle \cap\left\langle\nu_{d}(r)\right\rangle\right)$ are formed by a unique point $\mathrm{O}_{2}$ (resp., $\mathrm{O}_{3}$ ). Remark 3 gives that $O_{i} \in \mathbb{P}^{N}(\mathbb{R}), i=1,2, S_{\mathbb{C}, l}$ evinces $r_{\mathbb{C}}\left(O_{2}\right), S_{\mathbb{R}, l}$ evinces $r_{\mathbb{R}}\left(O_{2}\right)$, $S_{\mathbb{C}, r}$ evinces $r_{\mathbb{C}}\left(\mathrm{O}_{3}\right)$, and $S_{\mathbb{R}, r}$ evinces $r_{\mathbb{R}}\left(\mathrm{O}_{3}\right)$. The hypotheses of the case (1.2.3) coincide with (c)-(ii) of the statement of the theorem, while (15) gives also property (c)-(i). Moreover,
$\mathrm{O}_{2}$ and $\mathrm{O}_{3}$ defined above coincide with $\mathrm{O}_{l}$ and $\mathrm{O}_{r}$ in (c)(iv) of Theorem 1; therefore, we have also proved case (c) of Theorem 1.

(2) Now assume the existence of a conic $C \subset \mathbb{P}^{m}$ such that

$$
\operatorname{deg}\left(S_{\mathbb{C}} \cup S_{\mathbb{R}}\right)_{C} \geq 2 d+2
$$

Since $\sharp\left(S_{\mathbb{C}} \cup S_{\mathbb{R}}\right)_{C} \leq 3 d-1-2 d-2 \leq d-1$, we have $h^{1}\left(\mathscr{I}_{\left(S_{\mathbb{C}} \cup S_{\mathbb{R}}\right)_{\hat{C}}}(d-2)\right)=0$. By Lemma 4 we have

$$
S_{\mathbb{C}, \widehat{C}}=S_{\mathbb{R}, \widehat{C}}
$$

the set $\left\langle\{P\} \cup v_{d}\left(S_{\mathbb{C}, \hat{C}}\right)\right\rangle \cap\left\langle v_{d}(\langle C\rangle)\right\rangle$ is a single point:

$$
P^{\prime}:=\left\langle\{P\} \cup v_{d}\left(S_{\mathbb{C}, \widehat{C}}\right)\right\rangle \cap\left\langle v_{d}(\langle C\rangle)\right\rangle
$$

and $S_{\mathbb{C}, C}$ evinces $r_{\mathbb{C}}\left(P^{\prime}\right)$. Moreover, if $C$ is defined over $\mathbb{R}$, then $P^{\prime} \in \mathbb{P}^{N}(\mathbb{R})$ and $S_{\mathbb{R}, C}$ evinces $r_{\mathbb{R}}\left(P^{\prime}\right)$. Hence, $\sharp\left(S_{\mathbb{C}, C}\right)<\sharp\left(S_{\mathbb{C}} \cap\right.$ $\left.S_{\mathbb{R}}\right)$.

(2.1) Assume that $C$ is smooth. Therefore, (24) proves (b)(i) of the statement of the theorem in the case where $C$ is smooth. Since the reduced case is proved above (immediately after the displayed formula (15)), we have concluded the proof of (b)-(i).

Moreover, the hypothesis (23) coincides with (b)-(iii) of the statement of the theorem since $\sharp\left(S_{\mathbb{C}, C}\right)$ is obviously strictly smaller than $\sharp\left(S_{\mathbb{R}, C}\right)$. This concludes (b)-(iii).

The fact that $\sharp\left(S_{\mathbb{C}, C}\right)<\sharp\left(S_{\mathbb{R}, C}\right)$ also implies that $\sharp\left(S_{\mathbb{R}, C}\right) \geq$ 5. Since each point of $S_{\mathbb{R}}$ is real, $C$ is real. Remark 3 gives that $\nu_{d}\left(S_{\mathbb{R}, C}\right)$ evinces $r_{\mathbb{R}}\left(P^{\prime}\right)$. Since $S_{\mathbb{R}, C} \subset C, S_{\mathbb{R}, C}$ also evinces the real symmetric tensor rank of $P^{\prime}$ with respect to the degree $2 d$ rational normal curve $v_{d}(C)$. The point $P^{\prime}$ defined in (25) plays the role of the point $P_{C}$ appearing in (b)-(ii) of the statement of the theorem. Therefore, we have just proved (b)-

(ii) of Theorem 1.

We treat the case (2.2) below for the sake of completeness, but we can observe that this concludes the proof of Theorem 1 .

(2.2) Assume that $C$ is reducible, say $C=L_{1} \cup L_{2}$ with $L_{1}$ and $L_{2}$ lines and $\sharp\left(\left(S_{\mathbb{C}} \cup S_{\mathbb{R}}\right)_{L_{1}}\right) \geq \sharp\left(\left(S_{\mathbb{C}} \cap S_{\mathbb{R}}\right)_{L_{2}}\right)$. If $\sharp\left(\left(S_{\mathbb{C}} \cup\right.\right.$ $\left.\left.S_{\mathbb{R}}\right) \cap\left(L_{2} \backslash L_{2} \cap L_{1}\right)\right) \leq d$, then we proved in step (1) that we are in case (a) with respect to the line $L_{1}$. Hence, we may assume $\sharp\left(\left(S_{\mathbb{C}} \cup S_{\mathbb{R}}\right) \cap\left(L_{2} \backslash L_{2} \cap L_{1}\right)\right) \geq d+1$. Thus, even condition (b)-(iv) is satisfied as already remarked above after the displayed formula (15).

\section{Acknowledgments}

The authors were partially supported by CIRM of FBK Trento (Italy), Project Galaad of INRIA Sophia Antipolis Méditerranée (France), Institut Mittag-Leffler (Sweden), Marie Curie: Promoting science (FP7-PEOPLE-2009-IEF), MIUR, and GNSAGA of INdAM (Italy).

\section{References}

[1] L. De Lathauwer and J. Castaing, "Tensor-based techniques for the blind separation of DS-CDMA signals," Signal Processing, vol. 87, no. 2, pp. 322-336, 2007. 
[2] P. Comon, "Independent component analysis," in Higher Order Statistics, J.-L. Lacoume, Ed., pp. 29-38, Elsevier, London, UK, 1992.

[3] V. Strassen, "Rank and optimal computation of generic tensors," Linear Algebra and its Applications, vol. 52-53, pp. 645-685, 1983.

[4] L. G. Valiant, "Quantum computers that can be simulated classically in polynomial time," in Proceedings of the 33rd Annual ACM Symposium on Theory of Computing, pp. 114-123, ACM, New York, NY, USA, 2001.

[5] J. Eisert and D. Gross, "Multiparticle entanglement," in Physics Textbook, D. Bruß and G. Leuchs, Eds., Lectures on quantum information, pp. 237-252, Wiley-VCH, Weinheim, Germany, 2007.

[6] A. Bernardi and I. Carusotto, "Algebraic geometry tools for the study of entanglement: an application to spin squeezed states," Journal of Physics A, vol. 45, no. 10, Article ID 105304, 13 pages, 2012.

[7] E. S. Allman and J. A. Rhodes, "Phylogenetic ideals and varieties for the general Markov model," Advances in Applied Mathematics, vol. 40, no. 2, pp. 127-148, 2008.

[8] J. M. Landsberg, Tensors: Geometry and Applications, vol. 128 of Graduate Studies in Mathematics, American Mathematical Society, Providence, RI, USA, 2012.

[9] P. Comon and G. Ottaviani, "On the typical rank of real binary forms," Linear and Multilinear Algebra, vol. 60, no. 6, pp. 657667, 2012.

[10] A. Causa and R. Re, "On the maximum rank of a real binary form," Annali di Matematica Pura ed Applicata, vol. 190, no. 1, pp. 55-59, 2011.

[11] E. Ballico, "On the typical rank of real bivariate polynomials," . In press, http://arxiv.org/abs/1204.3161.

[12] G. Blekherman, “Typical real ranks of binary forms," . In press, http://arxiv.org/abs/1205.3257.

[13] E. Ballico and A. Bernardi, "A partial stratification of secant varieties of Veronese varieties via curvilinear subschemes," Sarajevo Journal of Mathematics, vol. 8, no. 1, pp. 33-52, 2012.

[14] E. Ballico and A. Bernardi, "Decomposition of homogeneous polynomials with low rank," Mathematische Zeitschrift, vol. 271, no. 3-4, pp. 1141-1149, 2012.

[15] A. Couvreur, "The dual minimum distance of arbitrarydimensional algebraic-geometric codes," Journal of Algebra, vol. 350, pp. 84-107, 2012.

[16] A. Bernardi, A. Gimigliano, and M. Idà, "Computing symmetric rank for symmetric tensors," Journal of Symbolic Computation, vol. 46, no. 1, pp. 34-53, 2011. 

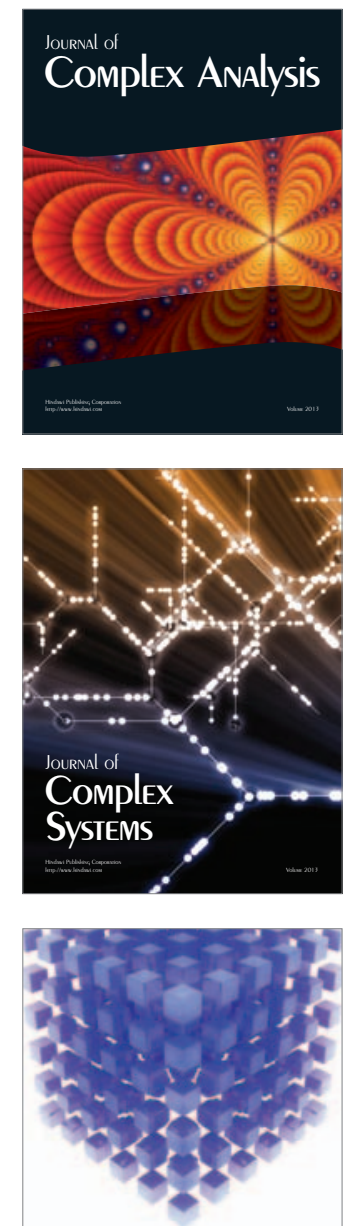

Mathematical Problems in ENGiNEERING
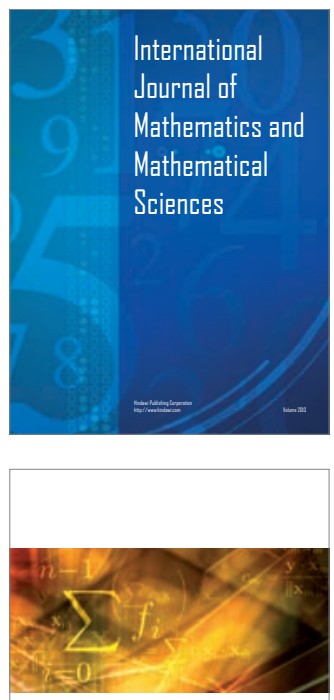

ISRN

Applied

Mathematics
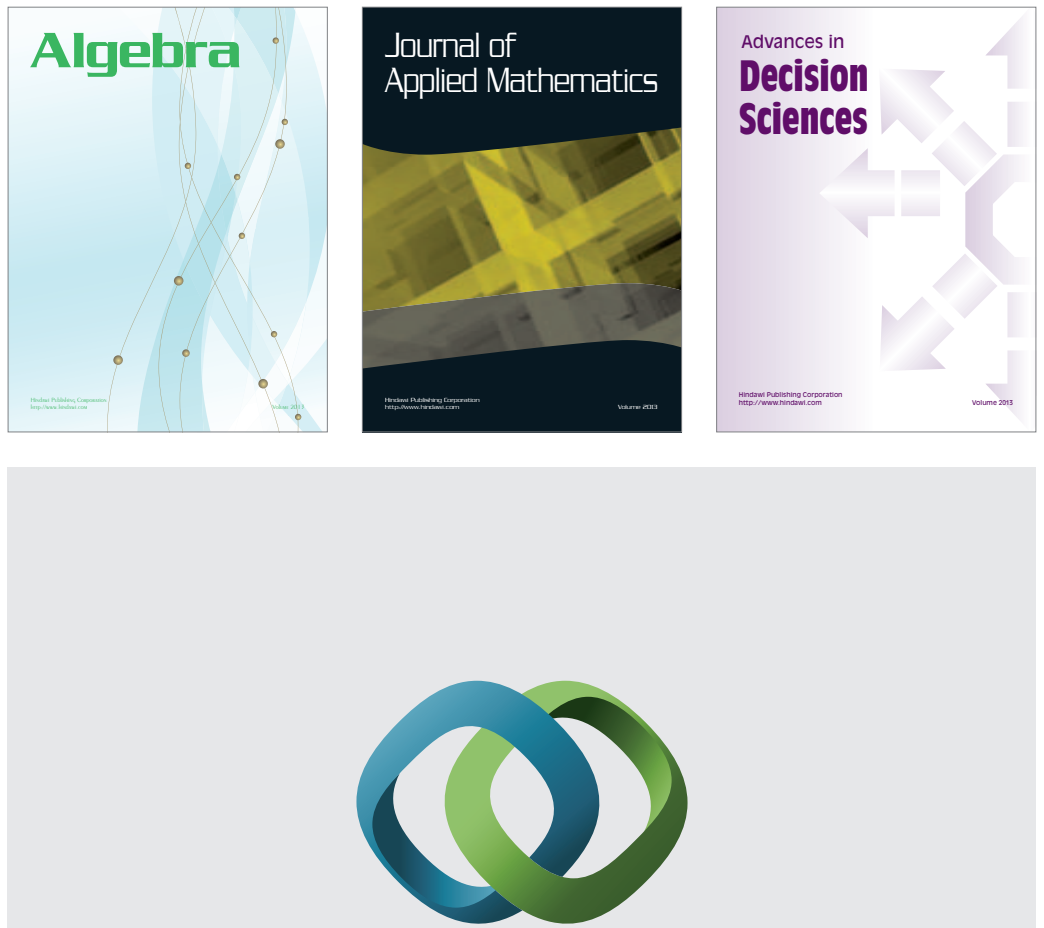

\section{Hindawi}

Submit your manuscripts at http://www.hindawi.com

Game Theory
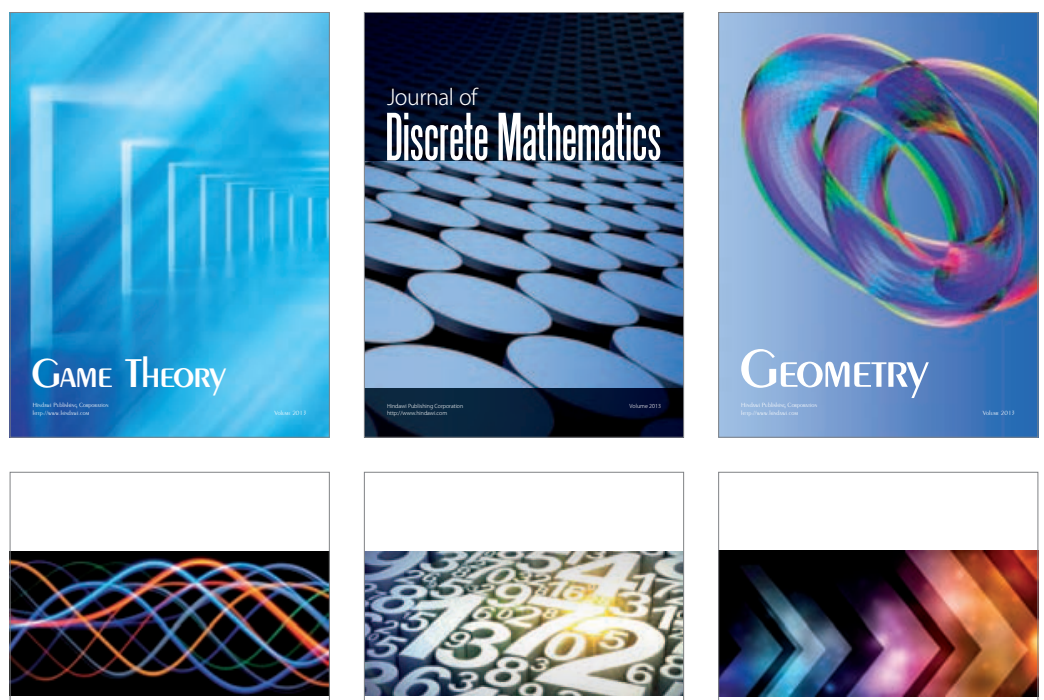

ISRN

Mathematical

Physics

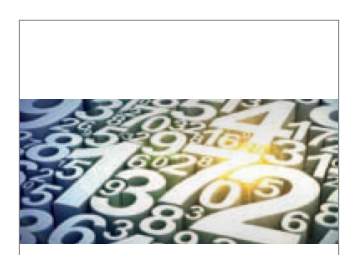

ISRN

Combinatorics

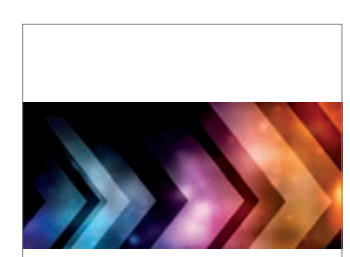

ISRN

Operations

Research
ISRN

Computational

Mathematics

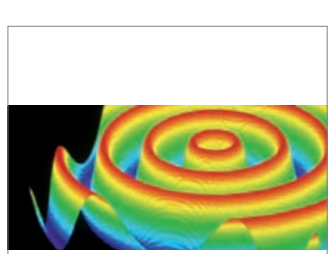

Abstract and

Applied Analysis
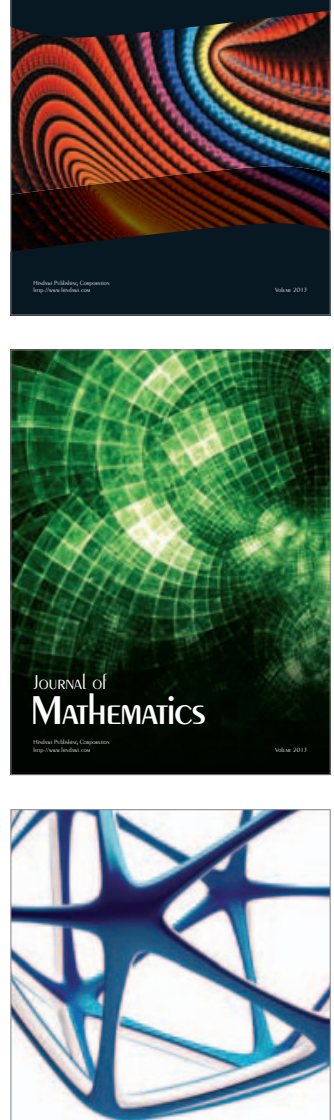

Ilownul of
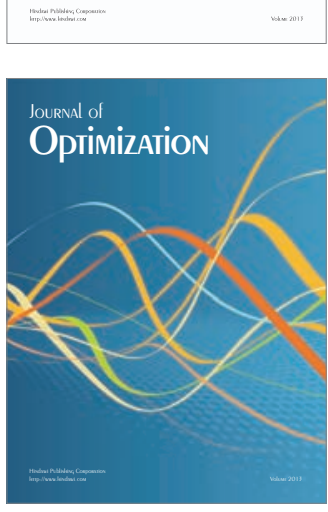

Mathematics 


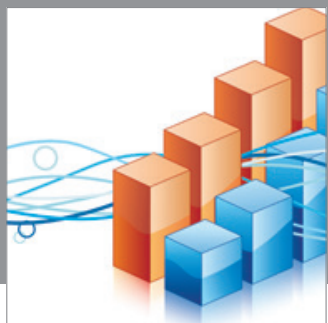

Advances in

Operations Research

mansans

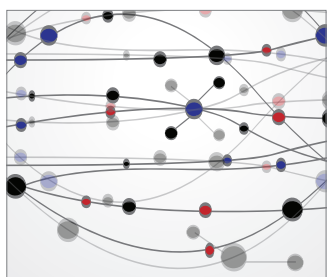

The Scientific World Journal
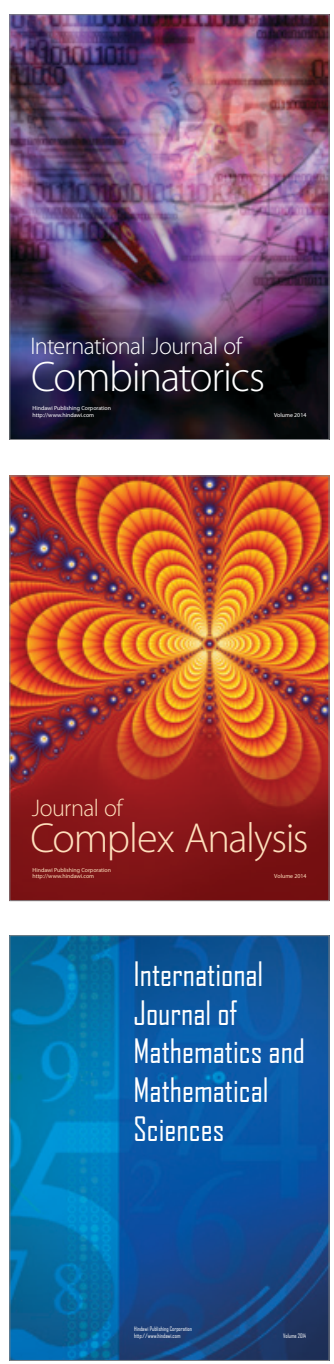
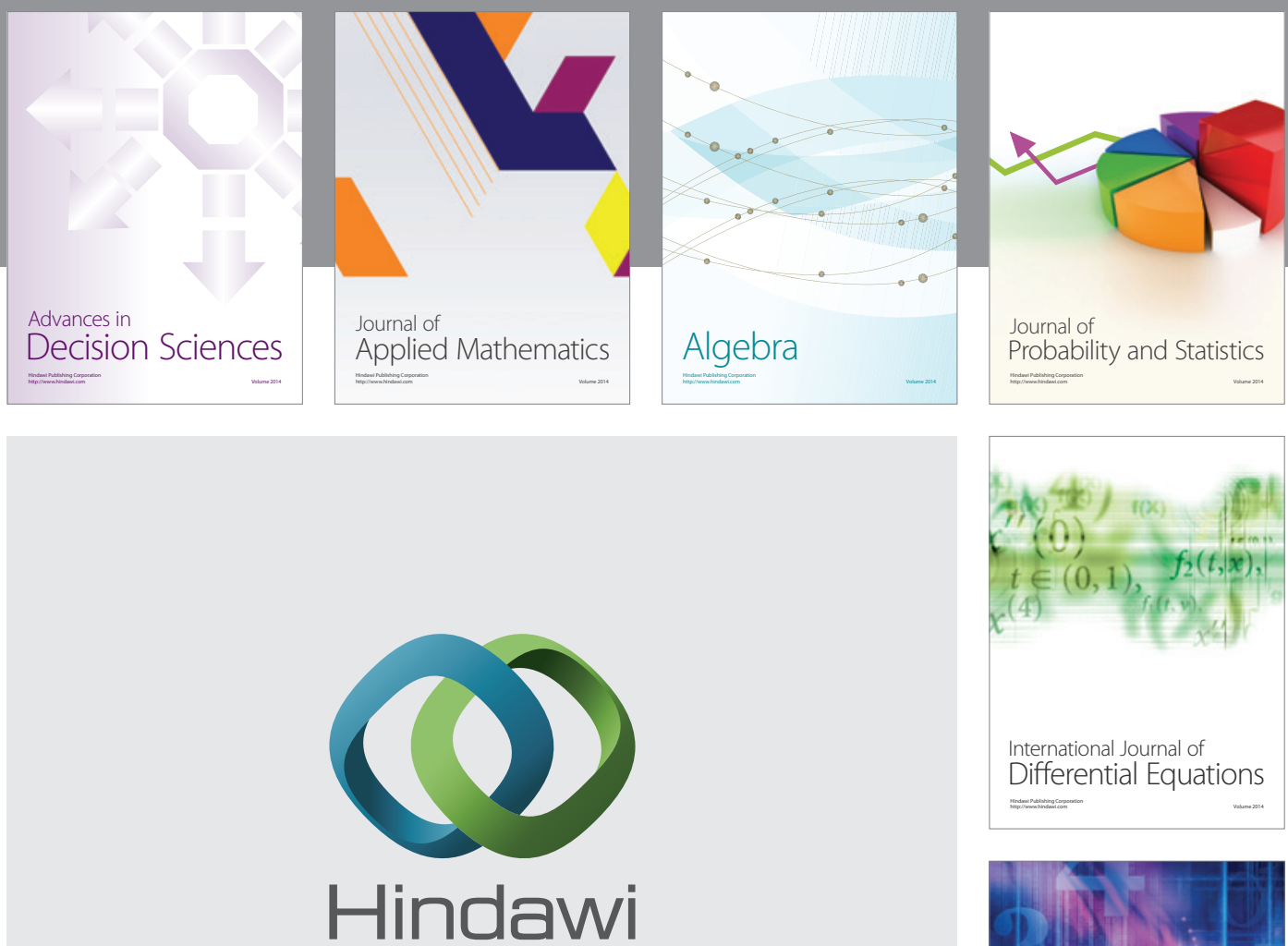

Submit your manuscripts at http://www.hindawi.com
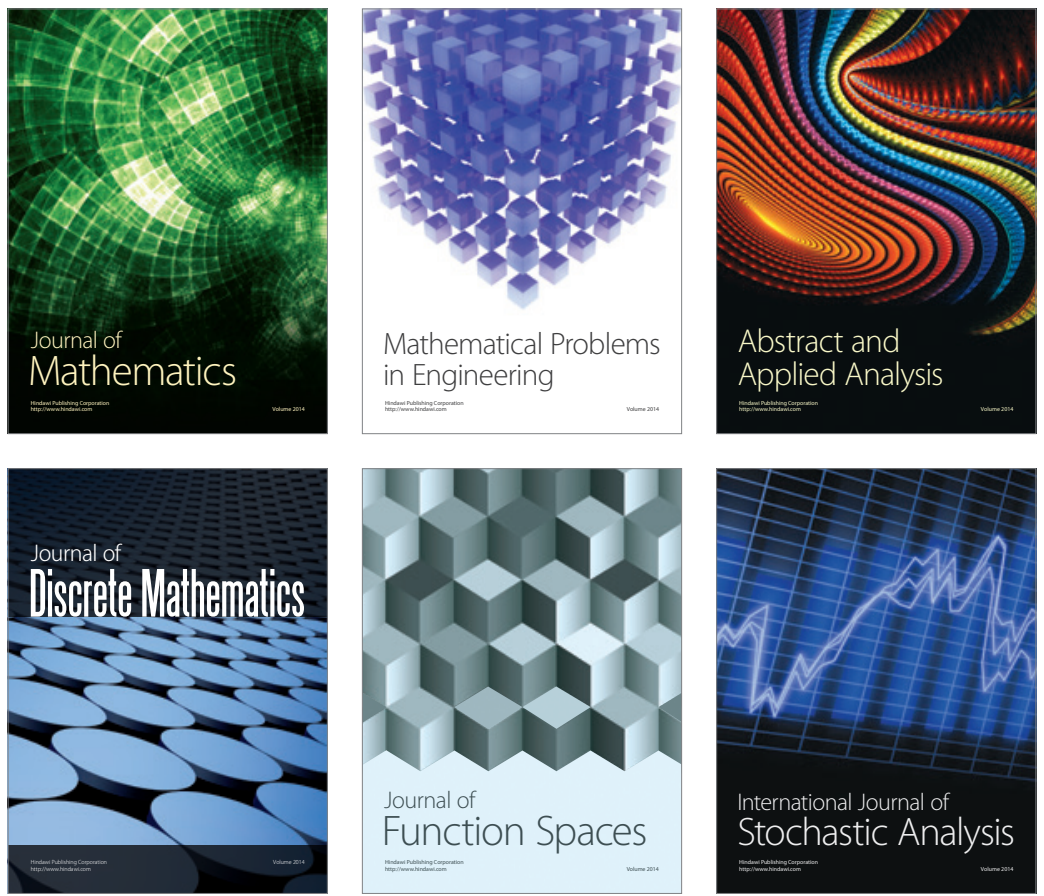

Journal of

Function Spaces

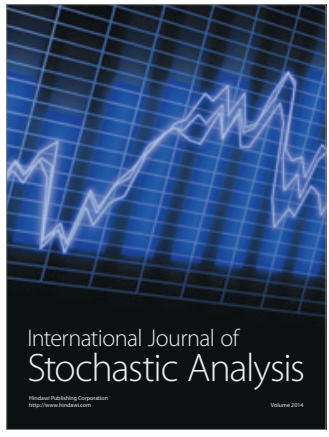

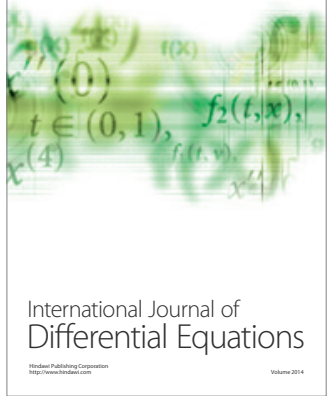
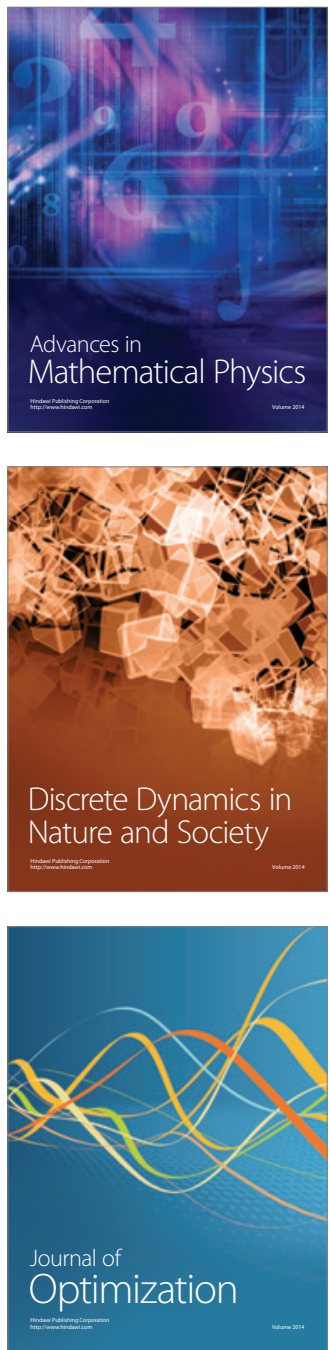